\title{
Gene Expression Profile Associated With Neural Tube Defects Among Infants Attending Muhimbili National Hospital And Muhimbili Orthopaedic Institute In Tanzania
}

\section{Mariam Sadallah}

Muhimbili University of Health and Allied Sciences

Evelyne Neema Assenga ( $\nabla$ eassenga@yahoo.com )

Muhimbili University of Health and Allied Sciences https://orcid.org/0000-0002-2281-2822

Charles Pallangyo

Muhimbili University of Health and Allied Sciences

Karim Manji

Muhimbili University of Health and Allied Sciences

\section{Research article}

Keywords: Gene Expression Profile Associated With Neural Tube Defects Among Infants Attending Muhimbili National Hospital, Muhimbili Orthopaedic Institute In Tanzania

Posted Date: March 13th, 2020

DOI: https://doi.org/10.21203/rs.3.rs-17225/v1

License: (9) This work is licensed under a Creative Commons Attribution 4.0 International License.

Read Full License 


\section{Abstract}

Background: Neural tube defects (NTDs) are severe congenital anomalies of the central nervous system. The specific cause is not known, though several factors including gene variants involving the folate metabolism have been implicated in the etiology. This study aimed at identifying the gene expression profile of selected genes known to be associated with NTDs among infants attending Muhimbili National Hospital (MNH) and Muhimbili Orthopaedic Institute (MOI).

Methodology: We conducted a matched case control study involving infants who were attending $\mathrm{MNH}$ and $\mathrm{MOI}$ during 6 months of the study period. Each case was matched with two controls by gestational age, sex and birth weight. Whole blood samples were collected from all study participants for genetic analysis. Total RNA was isolated using Qiagen RNA blood mini kit, and reversed transcribed into complementary DNA (cDNA) using Super Script II Reverse Transcriptase cDNA Synthesis kit. Real-time polymerase chain reaction was performed on extracted cDNA by a Light Thermal Cycler 480 machine using specific primers for studied genes to determine their expression levels. Results were analysed by GraphPad Prism 5Software, using Student t-test, and Bonferroni post hoc statistical tests. A p value of < 0.05 was considered to be statistically significant.

Results: The study recruited 50 cases and 100 controls. Among eight studied genes, we found significantly low expression of Methylenetetrahydrofolate reductase [MTHFR] gene among cases than controls $(p=0.006)$. Expression of others genes were having variations and expressed at very low levels in both cases and controls.

Conclusion: A low expression level of MTHFR gene was a significant risk factor for the occurrence of NTDs amongst infants attending $\mathrm{MNH}$ and $\mathrm{MOI}$.

Recommendation: We recommend gene expression analysis to be done on mothers of infants with NTDs to identify those at risk; and further mutational analysis to be done to enhance genetic counseling and therefore to prevent recurrence of NTDs.

\section{Introduction}

Neural tube defects (NTDs) are congenital malformations of the central nervous system including: the brain, spinal cord, and skull. These result from failure of the neural tube to close properly during fetal embryogenesis around the third and fourth week of conception(1). Globally, 300,000 babies are born with NTDs per year(2), with the prevalence ranging from 0.2 to 10 per 1000 live births in specific geographical locations(3). In Tanzania, in the study done by Kishimba et al in 2012,NTDs were reported to have the highest incidence of 9 per 10,000 live births among selected external structural defects.(4).

The majority of NTDs have unknown etiology, involving complex interactions of genetic, nutritional and environmental factors(5). Studies have shown that genetic mutations involving the folate metabolism and planar cell polarity (PCP) signaling pathways may be associated with an increased risk of NTDs(6). Gene variants that reduce the 'efficiency' of folate one-carbon metabolism have also been shown to 
increase the risk of NTDs. The Methylenetetrahydrofolate reductase (MTHFR) polymorphism C677T (rs1801133) has also been found to be associated with a 1.8-fold increased risk of NTDs, although this predisposition was detectable only in non-Hispanic populations(7). Similarly, in a study done in the Irish population, Kirke et al also found that both the homozygous (TT) and heterozygous (CT) genotypes for MTHFR C677T are associated with an increased risk of NTDs (odds ratio of 1.52 and 2.56 respectively) (8).In contrast, Hayati et al found no association between MTHFR C677T gene and NTDs in the Malaysian population as the homozygous genotype was absent in both cases and control groups(9). Another significant risk factor detected is the Methylenetetrahydrofolate dehydrogenase (MTHFD-1) R653Q variant (rs2236225), a trifunctional enzyme that catalyses the conversion of tetrahydrofolate to 5,10-methylenetetrahydrofolate(10). Polymorphism in the Methylenetetrahydrofolate dehydrogenase-1like(MTHFD-1-L), the gene for mitochondrial 10-formyl-tetrahydrofolate synthetase is also associated with an increased risk of NTDs(11).

Other genes such as Aminomethyltransferase (AMT) and Glycine dehydrogenase (GLDC) that encode enzymes of the glycine cleavage system have also been found to harbour a number of missense mutations resulting into NTDs. In the study done by Narisawa et al in 2011, two AMT gene variants c.589G $>C(D 197 H)$ and $c .850 G>C(V 284 \mathrm{~L})$ were identified in anencephaly and spinal bifida patients respectively, but they were absent in controls(12).

Planar cell polarity (PCP) genes such as VANGL1\&2,Dvl2\&Dvl3which are essential for proper gastrulation in the neural tube formation in humans, have also been implicated as predisposing factors of isolated non-syndromic NTDs. Lei et al, detected 3 missense mutations in VANGL 2 (S84F (737C $\rightarrow$ T), R353C $(1543 \mathrm{C} \rightarrow \mathrm{T})$ and F437S $(1796 \mathrm{~T} \rightarrow \mathrm{C})$ from studying 163 stillborn babies and miscarried Chinese fetuses with NTDs, and these mutations were found to be absent in the control group(13). Similarly, Kibar et al identified three Italian patients with sporadic and familial NTDs to have missense mutations in VANGL1(V239l, R274Q, and M328T) and these mutations were not identified in controls(14). Correspondingly, in a genetic study of a cohort of 473 NTD patients, De Marco et al found 8 rare mutations (5 in Dvl2 and 3 in Dvl3) in eight patients which were not present in the controls(15). Therefore, the evidence is highly suggestive of a genetic component as a contributory risk factor for NTDs. There is limited data on genetic etiology of NTDs in sub Saharan Africa of which Tanzania is part. Therefore, we conducted this study to determine gene expression profile for eight genes which have been implicated in NTDs causation from previous studies. The aim was to determine the genetic contribution to NTDs in our setting, which may serve as a basis for genetic counseling of couples, so as to reduce the incidence of NTDs.

\section{Materials And Methods}

A hospital-based matched case control study was conducted over a period of six months from November 2016 to April 2017 at the Muhimbili National Hospital and Muhimbili Orthopaedic Institute in, Dares Salaam Tanzania. All infants who were receiving neonatal, general paediatrics, and neurosurgical services, during the study period were eligible. 
Sampling procedure and sample size: A minimum of 50 cases and 100 controls were enrolled. A case was defined as infant with any type of NTDs and controls were those without detectable birth defects who met matching criteria. Consecutive sampling was used to enroll the cases until the sample size was reached. Two controls who matched a case by gestational age, birth weight and sex and who met the inclusion criteria were also selected using consecutive sampling among infants attending $\mathrm{MNH} / \mathrm{MOI}$ who had no obvious birth defects. Infants who met inclusion criteria but were very sick or whose mother did not consent were excluded.

Outcome variable: In this study, the dependent variable was the presence of the NTDs among the infants whilst for the independent variables; we selected eight genes which have been implicated from previous studies in NTDs etiology as shown in the Table 1.

Blood collection and laboratory procedures: We collected whole blood samples from all infants in the study for genetic analysis at MUHAS Genetic Laboratory. Blood samples were taken under aseptic technique using a 2 milliliter syringe and 1 milliliter volume of blood was collected into an EDTA vacutainer tube. The vacutainer were clearly labeled with the study identification number, time and date when the sample was drawn and sent to the laboratory in a cooler box within 2 hours of drawing the blood. In the laboratory, the samples were stored at $-80^{\circ} \mathrm{C}$ freezer to ensure preservation of genetic materials before analysis.

RNA extraction and RT-PCR analysis: Total RNA for all 150 infants were prepared from the blood samples using QIAamp RNA Blood Mini Kit (Qiagen) following the manufacturer's recommendations, $1 \mu \mathrm{g}$ of the total RNA was reverse transcribed into cDNA using Super Script II Reverse Transcriptase First-Strand cDNA Synthesis kit in accordance to the manufacturer's protocol (Invitrogen). A real-time polymerase chain reaction(RT-PCR) was performed on extracted cDNA by a Light Cycler 480 Real Time PCR system (Roche) according to the manufacturer's protocol using primers indicated in the Table 1, and 2x SYBR Green Master Mix (Roche). GAPDH was used as the house-keeping gene and the results were analyzed using the delta-delta $\mathrm{Ct}$ method $\left(2-{ }^{\Delta} \mathrm{C}\right.$ Ct method) and presented as relative gene expressions(16).The PCR reaction was performed using standard RT-PCR conditions: $50^{\circ} \mathrm{C}$ for 2 minutes, $95^{\circ} \mathrm{C}$ for 10 minutes, followed by 40 cycles of $95^{\circ} \mathrm{C}$ for 30 seconds and $60^{\circ} \mathrm{C}$ for 1 minute. 
Data analysis:Statistical analysis was performed using GraphPad Prism 5 (GraphPad Software, La Jolla, $\mathrm{CA}$ ) using the standard two-tailed Student's t-test for two data sets and ANOVA followed by Bonferroni post hoc tests for multiple data sets. A p-value of $<0.05$ was considered to be statistically significant.

\section{Results}

Socio- demographic characteristics of infants in the study population

A total of 50 cases and 100 controls participated in this study. Most of the infants were $<1$ month old ( $74 \%)$ with almost equal male to female ratio ( $52 \%$ male versus $48 \%$ female). The mean age of the participants was $1.78 \pm 3.2$ months with an age range of 1 to 11 months. Majority of the infants (92\%) were delivered at term, with $66 \%$ having normal birth weights as shown in Table 2.

Gene expression profile for eight (8) genes analyzed in the study population

We included eight genes of folate and planar cell polarity pathways which were selected from previously published NTDs researches as shown in the Table 1. After gene expression analysis, we found that there was a significantly low level of expression of the MTHFR gene amongst the NTDs cases than amongst the controls $(p=0.006)$. On the other hand, there were a lot of variations in the expression levels of other genes and these were expressed at very low levels in both controls and cases as shown in Figure 1.

\section{Discussion}

This study was conducted to determine the gene expression profile associated with NTDs among infants attending $\mathrm{MNH}$ and $\mathrm{MOI}$. Findings revealed a significant low expression of MTHFR gene among cases than controls.

NTDs result from failure of closure of the neural tube during fetal embryogenesis. Evidence from studies done in different population groups strongly suggest that MTHFR polymorphism (MTHFR C677T) is associated with an increased risk of NTDs $(7,8)$. In this study, DNA sequencing was not done, which is required for detecting specific gene mutation. However, the significantly low expression of MTHFR gene in the cases group could suggest the possibility of having variants with low enzyme activity amongst the cases with NTDs. The MTHFR gene plays a key role in folate and methylation cycles which are required for essential processes including; DNA synthesis, DNA/protein methylation, cell division, and tissue growth; especially in the rapidly developing cells(5). Thus, a defective gene variant could result in an impaired DNA synthesis or DNA methylation involved in the neurulation process which is essential during neural tube closure. 
The study found no association of NTDs and the expression levels of other genes such as VANGL 1, VANGL2,DV/2 and DV/3.This is contrary to the studies done in China and Italy, which found a significant association between the incidence of NTDs and other genes in addition to MTHFR gene(10-15). This could be partly explained by the fact that none of these studies were done in Africa and also the other studies included aborted fetuses. Therefore, there is the likelihood that, gene expressions can be influenced by genetic diversity among different population groups; as well as specific fetal developmental stages.

In this study, due to logistic reasons, DNA sequencing which is required for identifying specific genetic mutation/polymorphism could not be performed. Therefore we cannot link the gene expression profile with specific gene variant/mutation(s) which might be a risk factor among the NTDs cases.

\section{Conclusion And Recommendation}

In conclusion, our findings suggesting that a low expression level of MTHFR gene was a significant risk factor associated with NTDs amongst infants attending $\mathrm{MNH}$ and MOI. .

We recommend gene expression analysis to be done on mothers of infants with NTDs to identify those at risk; and further mutational analysis to be done to enhance genetic counseling and therefore to prevent recurrence of NTDs.

\section{Abbreviations}

cDNA: Complimentary Deoxyribonucleic Acid I GAPDH: Glyceraldehyde-3-Phosphate Dehydrogenase | MTHFD: Methylenetetrahydrofolate dehydrogenase I MTHFD: Methylenetetrahydrofolate reductase I MNH: Muhimbili National Hospital | MOI: Muhimbili Orthopaedic Institute | MUHAS: Muhimbili University of Health and Allied Sciences |NTDs: Neural Tube Defects | PCP: Planar Cell Polarity | RNA: Ribonucleic Acid | RT-PCR:' Real- time Polymerase Chain Reaction |

\section{Declarations}

\section{Acknowledgement}

We would like to give special thanks to the mothers and their infants with NTDs who participated in this research therefore helping in the realisation of the project. We would also like to thank the MUHAS Genetic Laboratory for hosting this research.

\section{Authors' contribution}


SM, AEN and PC conceived and designed the study. SM and PC jointly analysed the data, SM and AEN developed the paper and contributed to the writing of the manuscript and all authors reviewed the final manuscript.

\section{Competing interest}

The authors declare that they have no competing interests.

\section{Ethical approval and consent to participate}

Ethical clearance was obtained from the Institutional Review Board of Muhimbili University of Health and Allied Sciences (Ref.No.MU/PGS/SAEC/Vol.XVII/ and permission was sort from the Muhimbili National Hospital (Ref.No.MU/PGS/SAEC/Vol.XVII/ and Muhimbili Orthopaedic Institute authorities. All mothers signed informed consent forms prior to recruitment and received counseling on prevention of the future NTDs. The results from the genetic analysis were given through mobile phones for those who could be accessed. However, the results have been stored in a password secured folder whereby mothers who may need their individual information in future can be informed.

\section{Data availability}

The database supporting the conclusion of this article is available with the author within reasonable request

\section{Funding}

The study was conducted as part fulfillment of a master of medicine degree by dissertation which was funded by the Ministry of Health and Social Welfare in Tanzania, an estimated USD 685 equivalent to Tanzania shillings 1,500,000.

\section{References}

1. Merriam-Webster. Birth defect [ homepage on the internet]. c2015 [cited 2016 Apr 27]. Available from: http://www.merriam-webster.com/dictionary/birth + defect.

2. Christianson A, Howson CP, Modell B. March of dimes global report on birth defects. 2006. Pages Retrieved from http://www. marchofdimes. com/downloads/Birth_Defects_Report-PF. pdf. 2016.

3. Salih MA, Murshid WR, Seidahmed MZ. Epidemiology, prenatal management, and prevention of neural tube defects. Saudi Medical Journal. 2014;35(Suppl 1):S15.

4. Kishimba RS, Mpembeni R, Mghamba JM, Goodman D, Valencia D. Birth prevalence of selected external structural birth defects at four hospitals in Dar es Salaam, Tanzania, 2011-2012. Journal of 
global health. 2015 Dec;5(2).

5. Imbard A, Benoist JF, Blom HJ. Neural tube defects, folic acid and methylation. International journal of environmental research and public health. 2013 Sep 17;10(9):4352-89.

6. Copp AJ, Stanier P, Greene ND. Neural tube defects: recent advances, unsolved questions, and controversies. The Lancet Neurology. 2013 Aug 31;12(8):799-810.

7. Zhang T, Lou J, Zhong R, Wu J, Zou L SY et al. Genetic variants in the folate pathway and the risk of neural tube defects: a meta-analysis of the published literature. PLos one. 2013 Apr 4;8(4):e59570.

8. Kirke PN, Mills JL, Molloy AM, Brody LC, O'leary VB, Daly L et al. Impact of the MTHFR C677T polymorphism on risk of neural tube defects: case-control study. Bmj. 2004 Jun 24;328(7455):15356.

9. Hayati AR, Zainal Al, Tan GC, Ong LC, Khoo TB. MTHFR C677T polymorphism as a risk factor of neural tube defects in Malay: A Case Control Study. 2008;63(5):379-83.

10. Brody LC, Conley M, Cox C, Kirke PN, McKeever MP, Mills JL et al. A polymorphism, R653Q, in the trifunctional enzyme methylenetetrahydrofolate dehydrogenase/formyltetrahydrofolate synthetase is a maternal genetic risk factor for neural tube defects: report of the Birth Defects.The American Journal of Human Genetics. 2002;Nov 30:71(5):1207-15.

11. Parle-McDermott A, Pangilinan F, O'brien KK, Mills JL, Magee AM, Troendle J et al. A common variant in MTHFD1L is associated with neural tube defects and mRNA splicing efficiency. Human mutation. 2009 Dec 1;30(12):1650-6.

12. Narisawa A,Komatsuzaki S,Kikuchi A,Niihori T,Aoki Y,FujiwaraK et al.Mutations in Genes Encoding the Glycine Cleavage System Predispose to Neural Tube Defects in Mice and Humans. Human Molecular Genetics,2011 Dec 13;21(7):1496-503.

13. Lei Y-P, Zhang T, Li H, Wu B-L, Jin L, Wang H-Y. VANGL2 mutations in human cranial neural-tube defects. N Engl J Med. 2010;362(23):2232-5.

14. Kibar Z, Torban E, McDearmid JR, Reynolds A, Berghout J, Mathieu M et al. Mutations in VANGL1 associated with neural-tube defects. New England Journal of Medicine. 2007 Apr 5;356(14):1432-7.

15. De Marco P, Merello E, Consales A, Piatelli G, Cama A, Kibar Z et al. Genetic analysis of disheveled 2 and disheveled 3 in human neural tube defects. Journal of Molecular Neuroscience. 2013 Mar 1;49(3):582-8.

16. Schmittgen TD, Livak KJ. Analysis of Relative Gene Expression Data Using Real-Time Quantitative PCR and the 2- $\Delta \Delta C T$ Method. 2001;402-8.

\section{Tables}

Table 1.Primer sequences used for RT PCR 


\begin{tabular}{|l|l|l|}
\hline Gene & Forward5'à3' & Reverse5'à3' \\
\hline$A M T$ & GTGTTGGATAATGCCCTGCT & ACCGAGATCTCCACACCATC \\
\hline$D V L-2$ & GCTTTTGCAGGTGAATGACA & GAGGAGGAACCTGGATAGGC \\
\hline$D V L-3$ & GCTAAATGGAACTGCGAAGG & CCGCTTGTGTCTTCTCATCA \\
\hline GAPDH** & GTCAGTGGTGGACCTGACCT & TGCTGTAGCCAAATTCGTTG \\
\hline $\boldsymbol{M T F H D - 1}$ & TTCATCCCATGCACACCCAA & ATGCATGGGTGCACCAACTA \\
\hline $\boldsymbol{M T F H D - 1 L}$ & GGACCCACTTTTGGAGTGAA & ATGTCCCCAGTCAGGTGAAG \\
\hline $\boldsymbol{M T F H R}$ & AACCTGCCACTCAGGTGTCTTG & TGACAGTTTGCTCCCCAGGCAC \\
\hline $\boldsymbol{V A N G L - 1}$ & TTACCTCCGATCCTGTGGAG & AGGCCTTCATCCACATTCAG \\
\hline $\boldsymbol{V A N G L - 2}$ & CCAAACAGTGGACATTGGTG & GACTGCAGCCTCATGACAAA \\
\hline
\end{tabular}

** GADPH [Glyceraldehyde-3-Phosphate Dehydrogenase] was used as an internal control to ensure similar amounts of cDNA in the PCR mix

Table 2: Socio - demographic characteristics of 150 infants attending MNH and MOI

\begin{tabular}{|c|c|c|c|}
\hline Child characteristic & Case $(\mathrm{N}=50)$ & Control(N=100) & Total $(\mathrm{N}=150)$ \\
\hline \multicolumn{4}{|l|}{ Gender } \\
\hline Male & $26(52.0 \%)$ & $52(52.0 \%)$ & 78 \\
\hline Female & $24(48.0 \%)$ & $48(48.0 \%)$ & 72 \\
\hline \multicolumn{4}{|l|}{ Gestational age } \\
\hline Preterm & $4(8.0 \%)$ & $8(8.0 \%)$ & 12 \\
\hline Term & $46(92.0 \%)$ & $92(92.0 \%)$ & 138 \\
\hline \multicolumn{4}{|l|}{ Birth weight(kgs) } \\
\hline$<2.5$ & $5(10.0 \%)$ & $10(10.0 \%)$ & 15 \\
\hline $2.5-3.5$ & $33(66.0 \%)$ & $66(66.0 \%)$ & 99 \\
\hline$>3.5$ & $12(24.0 \%)$ & $24(24.0 \%)$ & 36 \\
\hline Mean age(months) & $1.78 \pm 3.259$ & $1.78 \pm 3.243$ & \\
\hline
\end{tabular}

\section{Figures}



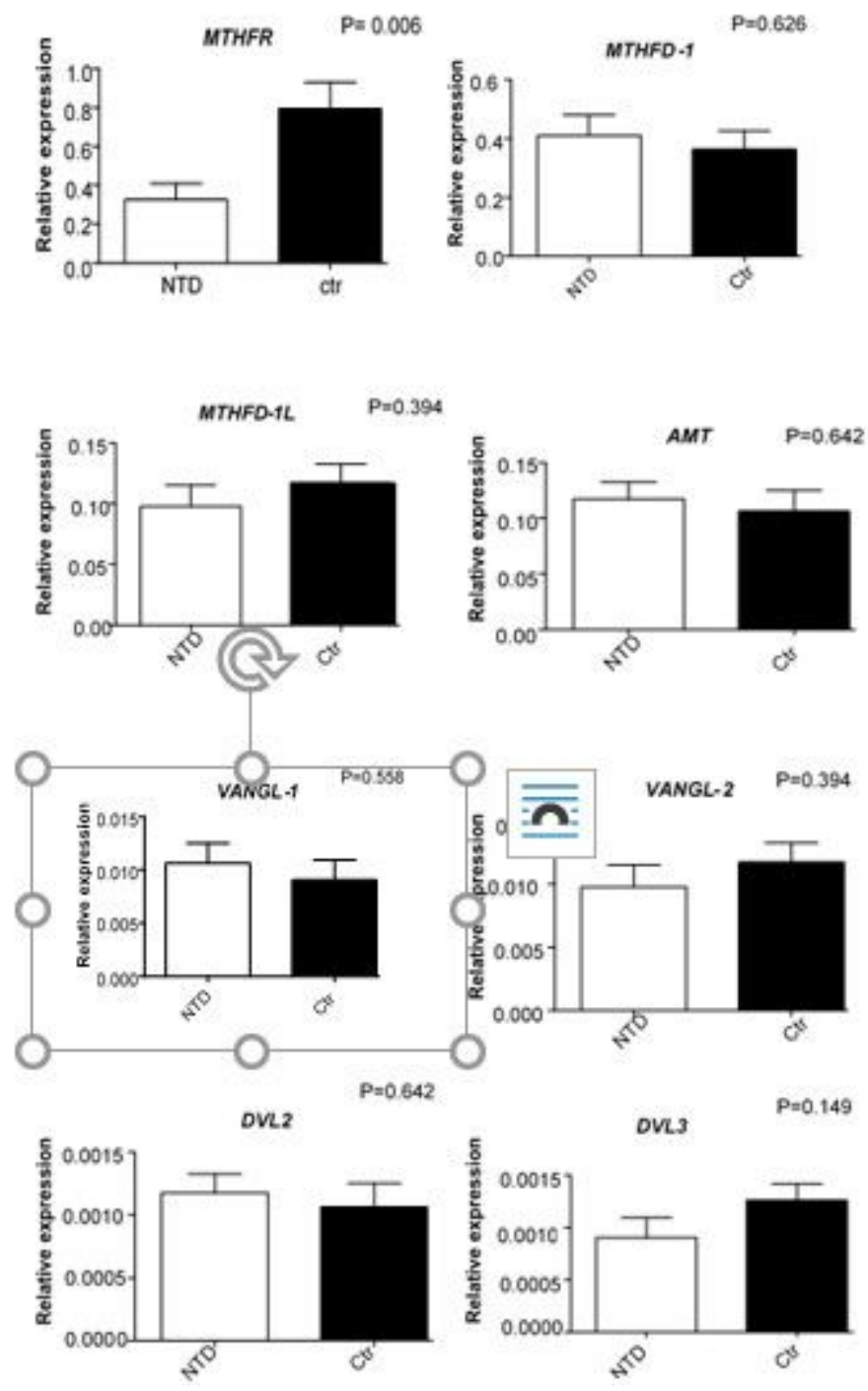

Figure 1

Relative gene expression profile for 8 selected genes analysed among 150 infants (NTD cases and controls) attending $\mathrm{MNH}$ and $\mathrm{MOI}$ 